\title{
Aging after spinal cord injury: an exploratory study
}

\author{
JS Krause*,1 \\ ${ }^{1}$ Crawford Research Institute, Atlanta, Georgia, USA
}

\begin{abstract}
Objective: To identify the relationship between two aspects of aging (age at injury onset and years since injury onset) with the post-injury prevalence of six classes of secondary conditions among a sample of participants with spinal cord injury (SCI).

Design: A cross-sectional study to identify the relationship between the two aging variables and secondary conditions after spinal cord injury.

Setting: A large southeastern rehabilitation hospital, with the collaboration of two midwestern hospitals.

Participants: Three hundred and forty-seven participants, all of whom had traumatic SCI of an average duration of 18.2 years.

Main outcome measures: The Secondary Conditions Questionnaire (SCQ), a 50-item selfreport screening measure for secondary conditions after SCI, was used to measure the postinjury prevalence of six classes of secondary conditions.

Results: There was a significantly greater odds of kidney stones, non-urinary related infections, and three types of musculoskeletal conditions (ie, curvature of the spine, contractures, and fractures) among participant cohorts who were 20-29 years post-injury and 30 or more years post-injury. The odds of heart problems and bowel obstructions were higher with a greater age at injury onset, whereas the odds of seven other conditions decreased among older cohorts at injury. Among these seven conditions, the most dramatic findings were for kidney stones, where individuals who were less than 18 at injury had over 30 times the odds of having had the condition since injury than those who were injured at 40 or older.

Conclusions: Although the study was cross-sectional in nature, the results point to secondary conditions which may be problematic with increasing years since injury, as well as those conditions which are more or less likely to occur among individuals injured at different points in their lives.
\end{abstract}

Spinal Cord (2000) 38, $77-83$

Keywords: SCI; Aging

\section{Introduction}

Spinal cord injury (SCI) occurs most frequently in young males who engage in high-risk activities. ${ }^{1}$ In fact, $4.2 \%$ of injuries occur in the $0-15$-year-old group, $56.4 \%$ in the $16-30$ group, $22.1 \%$ in the $31-45$ group, $10.3 \%$ in the $46-60$ group, and $7 \%$ for those 61 and older.

Although the sensory and motor limitations are of greatest concern immediately after the onset of SCI, there is also an increased long-term susceptibility to many types of secondary complications or secondary conditions (eg, pressure ulcers, urinary tract infections, and contractures). In contrast to the sensory and motor loss which are a direct result of SCI, these secondary conditions are generally indirectly related to SCI and do not occur to all individuals with SCI. For example, individuals with SCI are at greater risk for the development of pressure ulcers, due in part to the

*Correspondence: JS Krause, Crawford Research Institute, Shepherd Center, Atlanta, Georgia, USA losses of sensation and motor function associated with SCI (ie, the inability to feel the pressure ulcer developing or diminished ability to shift weight). ${ }^{2-6}$

There has been some epidemiological research that has helped to identify the prevalence of different secondary conditions after SCI, as well as their relationship to age and time since injury. A recent summary of data from the National Spinal Cord Injury Statistical Center (NSCISC) $^{1}$, a nationwide network of hospitals contributing patient data and other information to a common database, suggested that the rates of secondary conditions vary over the first 20 years after injury, the degree to which depends upon the condition under study. For example, pressure ulcers had their lowest prevalence during the first year after injury (15\%), but increased at a slow and steady rate thereafter $(26 \%$ in year 20$)$. A similar pattern was observed for urinary tract infections, which were reported in $62 \%$ of the cases in the first year after injury, but increased to nearly $95 \%$ by year 20 post- 
injury. In contrast, deep vein thromboses, pneumonia, and atelectasis rarely occurred after the rehabilitation period (rates remained under 3\% per year), despite occurring in between 12 to $20 \%$ of the cases during the first year post-injury. The primary limitations of the NSCISC data are high attrition and few available cases over 20 years post-injury.

The incidence of several types of secondary conditions was calculated in a study of follow-up diagnoses among 834 individuals from two hospitals in England. ${ }^{7}$ All SCIs occurred a minimum of 20 years earlier. Pressure ulcers and urinary tract infections were the most commonly occurring secondary conditions, with annual incidences of $23 \%$ and $20 \%$ respectively. Although not calculating odds ratios or significance tests, the authors noted four general patterns of relationships between secondary conditions with aging. First, several types of conditions that generally appear at early ages or soon after injury (eg, urinary retention, spasticity, and contractures) were unrelated to aging. Second, another set of conditions was more strongly related to age than to years since injury (eg, cardiac, pneumonia and other respiratory problems, and renal stones). A third set of conditions was generally unrelated to chronologic age, but was related to years since injury. These conditions included musculoskeletal problems (muscle or joint pain), and rectal abscess or bleeding. Lastly, a wide range of gastrointestinal problems, hemorrhoids, and nausea were most likely to occur among participants in both the oldest cohort (60 and older) and those with the most years post-injury (30 or more).

The study by Whiteneck and associates ${ }^{7}$ identified many important relationships between the two facets of aging and the annual incidence of secondary conditions after SCI. The inclusion of participants who had lived for 30 or more years since injury was particularly enlightening. However, there were several limitations in this study which may have affected the results, including no reporting of odds ratios or tests of statistical inference to identify the strengths or significance of age-related changes (these statistics were reported for mortality), reliance on follow-up diagnoses which may not have been sensitive to conditions of milder severity, and the confounding of chronologic age and years since injury.

This final limitation is inherent in aging studies, as chronologic age is a linear combination of age at injury onset and years since injury. Defining cohorts based on age at injury onset (rather than chronologic age) presents an alternative method that isolates the impact of aging up to the time of injury with that of aging after injury (ie, years since injury). Another methodological alternative to this study is to identify the relationship of these age related variables to the post-injury occurrence of secondary conditions (ie, whether the condition has occurred at any point since injury). This will identify points at which susceptibility to secondary conditions increases (eg, if the percentage of individuals who have ever had a fracture post-injury increases from $10 \%$ for those $10-19$ years post-injury to $35 \%$ for those $20-29$ years post-injury).

The purpose of this study was to identify the relationship between two aging parameters, years since injury onset and age at injury onset, with the post-injury prevalence of secondary conditions after SCI. Identifying differences in the prevalence of secondary conditions as a function of years since injury will identify conditions which appear only after individuals have lived for a certain period of time with SCI (ie, the impact of aging after or with SCI) and the number of years postinjury when the prevalence of the secondary condition increases. Identifying the prevalence of secondary conditions as a function of age at injury onset identifies conditions that are more or less likely to occur among people who were injured later in life. Conditions with a higher prevalence among those injured later in life would most likely be due to the adverse effects of age per $s e$, including pre-injury aging. In contrast, conditions with a decreasing or lower prevalence among people who were injured later in life would most likely be due to either the reduced number of years of life expectancy by virtue of being injured later in life (ie, they do not reach the same number of years post-injury as those who were injured at a younger age), or to a buffering effect of being older at injury.

\section{Methods}

\section{Participants}

All study participants met three inclusion criteria: (1) traumatic SCI, (2) a minimum of 2 years post-injury, and (3) 18 years of age or older at the time of the study. They were selected from outpatient files of two hospitals in the Midwestern United States, including a University hospital and a private rehabilitation hospital. All current study respondents had participated in a previous study of quality of life after SCI. Of the 435 respondents to the previous study, a total of 347 participated in the current study $(80 \%$ response rate). No data were collected regarding characteristics of non-respondents.

Eighty per cent of the participants were male and $97 \%$ were Caucasian. A total of $58 \%$ had cervical injuries. The average age of the participants was 25.7 years at injury and 43.9 years at the time of the study (an average of 18.2 years had passed since injury). The participants averaged 14.5 years of education. Just over half of the participants $(52 \%)$ were working at the time of the study.

\section{Instruments}

The Secondary Conditions Questionnaire (SCQ) was developed for this study to explore the prevalence of a wide range of secondary conditions. There are 50 items in the SCQ, each of which require individuals to indicate whether they have had a particular condition since injury and, if so, how recently. The SCQ does not elicit information on the number of times the condition 
occurred (eg, number of pressure ulcers) or the severity of the condition (eg, grade of pressure ulcer). Since the purpose of the current study was to identify the relationship between two aging components and the (at least) one-time prevalence of the conditions since injury, the responses were dichotomized, based on whether a condition had occurred at any time since injury.

The SCQ uses a self-report methodology that was not intended to generate clinical diagnoses. Rather, it was developed as a screening measure to identify the general symptoms, concerns, or problems that individuals perceive themselves to have or for which they have been treated by a health care professional. Each SCQ item relates to a symptom of a condition (eg, sweats or chills), a condition itself (eg, urinary tract infection), or a class of conditions (eg, heart problems). The conditions cover a wide range of possible long-term complications related to SCI, including both physiological and psychosocial conditions. The conditions were presented either by name alone (eg, cancer, or urinary tract infection), or by a brief description (eg, skin sore on ischium - where you sit). Psychosocial conditions were generally presented as one-word descriptors, such as depression, boredom, or stress. The 50 conditions were grouped into six general categories: (1) cardiopulmonary, (2) musculoskeletal, (3) genitourinary, (4) gastrointestinal, (5) skin, and (6) psychosocial. Scales were developed for each of the six primary classes of conditions. This classification of conditions is an extension of the five class scheme outlined by the Centers for Disease Control $(\mathrm{CDC})^{8}$, with the only differences being that a category for gastrointestinal conditions was included in this study, the CDC category 'cardiopulmonary' includes cardiovascular conditions, and the 'musculoskeletal' category is 'neuromusculoskeletal' in the CDC classification.

\section{Procedures}

All prospective participants were sent letters prior to study materials in order to explain the study and to solicit participation. The SCQ was sent $2-4$ weeks later, and a second mailing to all nonrespondents 4-6 weeks after the initial mailing. Follow-up phone calls were made if participants did not respond to either of the two mailings. Participants were offered a $\$ 12$ stipend and copies of the study results as inducements to participate.

\section{Analyses}

Participants were grouped into four cohorts based on age at injury onset: less than $18 \quad(n=60), 18-25$ $(n=171), 26-39 \quad(n=72)$, and 40 and older $(n=44)$. They were also grouped independently into four cohorts based on years since injury: less than 10 $(n=81), 10-19(n=114), 20-29(n=107), 30$ or more $(n=45)$. Whereas the years since injury cohorts represent 10-year intervals, the age at injury onset cohorts represent varying intervals due to the disproportionate percentage of individuals that are injured at younger ages ${ }^{6}$. This distribution of cases by age at injury onset and years since injury is not specific to the current study, as it is representative of the epidemiology of $\mathrm{SCI}^{6}$.

Odds ratios were calculated to identify differences in the odds of each condition occurring as a function of cohort variables (ie, age at injury onset and years since injury). Separate analyses were computed for age at injury onset and years since injury. The odds ratio is the ratio of the odds of an individual having a specific condition to the odds of an individual not having the condition. The odds is set to 1.0 for a base group, which in this study are participant cohorts who were injured at the youngest age (younger than 18), or those who had been injured the fewest years (ie, less than 10 years). Odds ratios greater than 1.0 indicate that individuals in that cohort had a greater odds of reporting the condition than the base cohort, whereas an odds ratio of less than 1.0 would indicate that cohort had a lower odds of reporting the condition. Odds ratios that are less than one can be converted to their equivalent reciprocal number greater than one to aid in interpretation (formula: [1/odds ratio]).

\section{Results}

\section{Years since injury}

Items Seven conditions increased among cohorts with greater years since injury (Table 1). Kidney stones showed the clearest increase among participants with greater years since injury. Compared with participants with less than 10 years post-injury, the odds of reporting kidney stones were over six times greater among individuals who had been injured $20-29$ years $(\mathrm{OR}=6.22)$ and over eight times greater among individuals who had been injured 30 or more years $(\mathrm{OR}=8.18)$. Although of a lesser magnitude, the odds of non-urinary related infections more than doubled among participants in the 20-29 group $(\mathrm{OR}=2.46)$ and more than tripled among participants who were 30 or more years post-injury $(\mathrm{OR}=3.26)$. Generally similar patterns were observed for three musculoskeletal conditions (curvature of the spine, fractures, and major contractures). The odds of each of these conditions were higher for the groups of participants that were $20-29$ and 30 or more years post-injury when compared with individuals who had been injured less than a decade (eg, for musculoskeletal conditions, those in the more years post-injury groups had at least twice the odds as those who were less than 10 years post-injury). The odds of curvature of the spine and major contractures were more than 2.5 times greater for participants in the two cohorts with the greatest number of years since injury. The odds for fractures for individuals in the cohort with 30 or more years postinjury was three times that of the cohort with the fewest 
years since injury. Two remaining conditions (rectal bleeding and alcohol or drug abuse) were significantly related to years since injury, although no clear trend was differentiated.

\section{Age at injury onset}

Items Fourteen of the 50 items were significantly related to age at injury onset (Table 2). The odds of two conditions were greater among those who were older at injury onset. Participants who were age 40 and older at injury had nearly six times the likelihood of reporting heart problems as participants who were injured prior to the age of $18(\mathrm{OR}=5.75)$. The odds of endorsing the item 'bowel obstruction' also increased with increasing age at injury onset. The odds increased significantly for participants aged $18-25$ at injury $(\mathrm{OR}=2.76)$ and those aged $26-39(\mathrm{OR}=2.98)$, reach-

Table 1 Percentage reporting secondary conditions as a function of years since injury

\begin{tabular}{|c|c|c|c|c|c|}
\hline Variable & $\begin{array}{l}\text { Percent- } \\
\text { age }\end{array}$ & $\begin{array}{l}\text { Odds } \\
\text { ratio }\end{array}$ & $\begin{array}{l}\text { Confidence } \\
\text { lower }\end{array}$ & $\begin{array}{l}\text { Interval } \\
\text { upper }\end{array}$ & Wald \\
\hline \multicolumn{5}{|c|}{ Kidney or bladder stones } & $41.85 * * *$ \\
\hline$<10$ & 15.0 & - & - & - & \\
\hline $10-19$ & 23.9 & 1.78 & 0.84 & 3.77 & \\
\hline $20-29$ & 52.3 & $6.22 * * *$ & 3.02 & 12.80 & \\
\hline $30+$ & 59.1 & $8.18 * * *$ & 3.47 & 19.31 & \\
\hline \multicolumn{5}{|c|}{ Infection other than UTI } & $11.08 * *$ \\
\hline$<10$ & 23.5 & - & - & - & \\
\hline $10-19$ & 35.1 & 1.76 & 0.93 & 3.35 & \\
\hline $20-29$ & 43.0 & 2.46 & 1.30 & 4.67 & \\
\hline $30+$ & 50.0 & $3.26 * *$ & 1.49 & 7.14 & \\
\hline \multicolumn{5}{|c|}{ Curvature of spine } & $14.60 * *$ \\
\hline$<10$ & 30.0 & - & - & - & \\
\hline $10-19$ & 35.7 & 1.30 & 0.70 & 2.40 & \\
\hline $20-29$ & 53.3 & $2.67 * *$ & 1.44 & 4.92 & \\
\hline $30+$ & 54.5 & $2.80 * *$ & 1.31 & 6.00 & \\
\hline \multicolumn{5}{|c|}{ Major broken bones } & $13.27 * *$ \\
\hline$<10$ & 18.5 & - & - & - & \\
\hline $10-19$ & 23.7 & 1.37 & 0.67 & 2.77 & \\
\hline $20-29$ & 38.7 & $2.78 * *$ & 1.40 & 5.50 & \\
\hline $30+$ & 40.9 & $3.05^{* *}$ & 1.34 & 6.93 & \\
\hline \multicolumn{5}{|c|}{ Major contractures } & $7.67 *$ \\
\hline$<10$ & 14.3 & - & - & - & \\
\hline $10-19$ & 23.0 & 1.79 & 0.83 & 3.89 & \\
\hline $20-29$ & 30.8 & $2.66 * *$ & 1.24 & 5.71 & \\
\hline $30+$ & 31.8 & $2.80^{*}$ & 1.14 & 6.88 & \\
\hline \multicolumn{5}{|c|}{ Rectal bleeding } & $10.02 *$ \\
\hline$<10$ & 49.4 & - & - & - & \\
\hline $10-19$ & 62.3 & 1.69 & 0.95 & 1.78 & \\
\hline $20-29$ & 44.9 & 0.83 & 0.47 & 1.78 & \\
\hline $30+$ & 38.6 & 0.65 & 0.26 & 1.36 & \\
\hline \multicolumn{5}{|c|}{ Drug abuse } & $12.56 * *$ \\
\hline$<10$ & 8.6 & - & - & - & \\
\hline $10-19$ & 24.8 & $3.48 * *$ & 1.44 & 8.44 & \\
\hline $20-29$ & 19.6 & $2.58 *$ & 1.04 & 6.41 & \\
\hline $30+$ & 4.5 & 0.50 & 0.10 & 2.54 & \\
\hline
\end{tabular}

$* P<0.05, * * P<0.01 . * * * P<0.001$ ing a high point with those who were oldest at injury $(\mathrm{OR}=4.42)$.

The odds of several secondary conditions were lower with increasing age at injury onset. Kidney stones were the secondary condition with the greatest decrease in prevalence based on age at injury onset. Compared with individuals who were injured prior to 18 , the odds of reporting a kidney stone at some time since injury dropped to 0.35 for individuals injured between the ages of 18 and 25 at injury, to 0.18 for those injured between the ages of 26 and 39, to 0.03 for persons who were injured at 40 and older (more than 30 times less likely to report the condition). Among skin related conditions, individuals who were oldest at injury reported a lower odds of both burns $(\mathrm{OR}=0.45)$ and pressure ulcers in areas other than the ischium, buttocks, hips, or coccyx $(\mathrm{OR}=0.34)$. Similarly, individuals who were oldest at injury had a lower odds of reporting sweats and chills $(\mathrm{OR}=0.34)$. Being older at injury was also associated with a lower odds of curvature of the spine, both among individuals who were age 26-39 at injury $(\mathrm{OR}=0.48)$ and those who were 40 or older at injury $(\mathrm{OR}=0.28)$. Three conditions were significantly related to age at onset but did not show a distinctive pattern of change (rectal bleeding, calcium deposits, and headaches).

Two psychosocial conditions were significantly related to age at injury onset. Compared with individuals who were injured prior to 18 , the odds of reporting prolonged stress were lower among individuals who were between ages 26 and 39 at injury $(\mathrm{OR}=0.38)$ and among individuals who were over 40 at injury $(\mathrm{OR}=0.23)$. Family conflicts were also lower among those in the oldest cohort $(\mathrm{OR}=0.29)$. Two conditions were significantly related to age at onset but did not show a distinctive pattern of change (boredom and sleep disorders).

\section{Discussion}

The purpose of this exploratory study was to generate self-report data on the relationship between aging and the post-injury occurrence of secondary conditions after SCI. The primary strengths of this study were the longevity of the participant sample, as many participants had lived for more than three decades with SCI, and the number and diversity of conditions investigated. The primary limitations related to the utilization of self-report data and the lack of measurement of severity or frequency of secondary conditions. The results of the study helped to identify relative risk of secondary conditions between different cohorts of participants based on age at injury and years since injury.

\section{Years since injury}

The conditions that were most highly correlated with years since injury generally reflected chronic muscu- 
Table 2 Percentage reporting secondary conditions as a function of age at injury onset

\begin{tabular}{|c|c|c|c|c|c|}
\hline Variable & $\begin{array}{l}\text { Percent- } \\
\text { age }\end{array}$ & $\begin{array}{l}\text { Odds } \\
\text { ratio }\end{array}$ & $\begin{array}{l}\text { Confidence } \\
\text { lower }\end{array}$ & $\begin{array}{l}\text { Interval } \\
\text { upper }\end{array}$ & Wald \\
\hline \multicolumn{5}{|c|}{ Heart problems } & $9.24 *$ \\
\hline$<18$ & 5.0 & - & - & - & \\
\hline $18-25$ & 10.6 & 2.25 & 0.64 & 7.91 & \\
\hline $26-39$ & 7.1 & 1.46 & 0.33 & 6.38 & \\
\hline $40+$ & 23.3 & $5.75 * *$ & 1.48 & 22.37 & \\
\hline \multicolumn{5}{|c|}{ Bowel obstruction } & $9.45^{*}$ \\
\hline$<18$ & 13.3 & - & - & - & \\
\hline $18-25$ & 29.8 & $2.76^{* *}$ & 1.2 & 6.22 & \\
\hline $26-39$ & 31.4 & $2.98 * *$ & 1.2 & 7.37 & \\
\hline $40+$ & 4.05 & $4.42 * *$ & 1.6 & 11.60 & \\
\hline
\end{tabular}

Kidney or bladder stones

$\begin{array}{lrrrr}<18 & 63.3 & - & - & - \\ 18-25 & 37.4 & 0.35^{* * *} & 0.19 & 0.64 \\ 26-39 & 23.9 & 0.18^{* * *} & 0.09 & 0.39 \\ 40+ & 4.9 & 0.03 * * * & 0.01 & 0.14\end{array}$

Burns below where you can feel $<18$

$18-25$ 59.3

$26-39$

65.5

$40+$

39.5

Other skin sores

$<18 \quad 50.0$

$18-25 \quad 54.4$

$26-39 \quad 50.7$

$40+\quad 25.6$

Sweats or chills

$<18 \quad 76.3$

$18-25 \quad 78.2$

$26-39 \quad 72.9$

$40+\quad 52.4$

Curvature of spine

$<18 \quad 55.9$

$18-25 \quad 42.9$

$26-39 \quad 38.0$

$40+$

26.2

Rectal bleeding

$<18 \quad 40.0$

$18-25 \quad 59.1$

26-39 47.9

$40+\quad 39.5$

Calcium deposits

$<18 \quad 25.0$

$18-25 \quad 24.1$

26-39 37.1

$40+$

10.0

Headaches

$<18$

$18-25$

53.3

$26-39$

$40+$

62.9

63.4

33.3

Stress

$<18$

$18-25$

26-29

84.7

75.9

67.6

55.8
1.30

1.21

$0.45^{*}$

0.71

0.59

1.19

1.03

$0.34 * *$

0.66

0.52

0.15

2.05

0.81

1.

0.55

$0.84 \quad 0.38$

$0.34 * * \quad 0.15$

0.59

0.48 *

$0.28 * *$

0.32

0.23

0.12

0.98

0.65

$2.16^{* * *}$

1.19

1.38

0.98

0.69

0.44

3.94

2.76

2.18

0.95

$\begin{array}{ll}0.77 & 0.83\end{array}$

0.48

0.10

1.89

3.79

1.09

1.49

1.51

$0.44 *$

0.82

0.82
0.75

0.19

$32.43 * * *$

.64
.39

.14

2.38

2.47

1.00

$10.65^{* *}$

$9.55^{*}$

2.26

1.86

0.80

$9.21 *$

$9.76^{*}$

$12.70^{* * *}$

2.69

3.05

0.99

$11.80^{* *}$

$\begin{array}{cc}-\overline{5} & - \\ 0.26\end{array}$

$0.38 * \quad 0.16$

$0.23 * * \quad 0.09$

1.25

0.89

2.19
$11.14^{* *}$ loskeletal conditions that, for the most part, pose no serious threats to longevity (eg, curvature of the spine, major contractures, and fractures). These conditions may, to varying degrees, be prevented with appropriate seating, equipment, and physical therapy. The development of kidney stones or infections present more severe threats to health. The dramatic increase in risk for kidney stones suggests that a great many people who have aged with SCI are living with this condition or with the residual impact of having been treated for this condition. Even though noninvasive, or less invasive surgical techniques have been developed in recent years, many people with SCI are living with scar tissue from earlier surgeries. This should be of note to health care professionals who work with people who have lived 20 or more years with SCI, due to diminished renal functioning.

At first glance, it is surprising that the post-injury prevalence of the majority of other conditions was not correlated with years since injury. However, there are several important explanations for this pattern of results. First, the cohort with the fewest years since injury ranged from $2-9$ years. The first 10 years after injury may be critical for the development of several types of secondary conditions, whereas conditions that do not occur during the first few years after SCI are not likely to occur at all. Psychosocial conditions appear to fit this pattern. Second, many conditions, such as urinary leakage and bowel accidents, are a reflection of the nature and severity of the injury itself. Although frequency of secondary conditions may change with aging (we can not determine this from the current data), the probability of their occurrence at some time after injury is not likely to be related to aging. Third, conditions such as pressure ulcers are preventable with appropriate management and, therefore, may be more highly correlated with personality or beliefs systems. Although aging may indeed affect these conditions, any adverse impact is most likely to

Table 2 (Continued)

\begin{tabular}{|c|c|c|c|c|c|}
\hline Variable & $\begin{array}{l}\text { Percent- } \\
\text { age }\end{array}$ & $\begin{array}{l}\text { Odds } \\
\text { ratio }\end{array}$ & $\begin{array}{l}\text { Confidence } \\
\text { lower }\end{array}$ & $\begin{array}{l}\text { Interval } \\
\text { upper }\end{array}$ & Wald \\
\hline \multicolumn{5}{|c|}{ Family conflicts } & $8.65^{*}$ \\
\hline$<18$ & 51.7 & - & - & - & \\
\hline $18-25$ & 47.3 & 0.84 & 0.47 & 0.52 & \\
\hline $26-29$ & 47.9 & 0.86 & 0.43 & 0.71 & \\
\hline $40+$ & 23.8 & $0.29 * *$ & 0.12 & 0.70 & \\
\hline \multicolumn{5}{|l|}{ Boredom } & $7.65^{*}$ \\
\hline$<18$ & 61.7 & - & - & - & \\
\hline $18-25$ & 63.3 & 1.07 & 0.58 & 1.97 & \\
\hline $26-29$ & 50.7 & 0.64 & 0.32 & 1.29 & \\
\hline $40+$ & 42.9 & 0.47 & 0.21 & 1.04 & \\
\hline \multicolumn{5}{|c|}{ Sleep disorders } & $8.46^{*}$ \\
\hline$<18$ & 45.0 & - & - & - & \\
\hline $18-25$ & 46.7 & 1.07 & 0.59 & 1.94 & \\
\hline $26-29$ & 66.2 & $2.39 *$ & 1.10 & 4.86 & \\
\hline $40+$ & 52.4 & 1.34 & 0.61 & 2.96 & \\
\hline
\end{tabular}


occur to individuals who are already at the greatest risk (ie, those who have already had sores). Therefore, it is possible that aging may impact reoccurrence of these types of conditions, even though participants who did not report these conditions during the first 10 years after injury reported little additional risk with age. Lastly, the data were cross-sectional in nature. It has been previously documented that individuals who are less well adjusted after SCI do not survive as long as those with superior adjustment patterns. ${ }^{9-11}$ Therefore, it is likely that individuals who survive past two or three decades after injury are those that avoid certain types of secondary conditions, including pressure ulcers, depression, alcohol abuse, and other types of preventable conditions.

\section{Age at injury onset}

Comparisons based on age at injury identified which conditions may be associated with aging per se (rather than with aging after SCI). The only two conditions in which there appeared to be greater susceptibility with an older onset of injury were heart problems and bowel obstruction (which, given the rate of occurrence, may have been interpreted by participants as constipation). Both these conditions probably reflect the impact of age per se, including aging prior to injury, rather than aging with SCI.

In contrast, conditions that were negatively correlated with age at onset were those that may simply never occur due to several factors. First, the late age at injury onset and associated fewer years of post-injury life expectancy may preclude musculoskeletal conditions such as curvature of the spine. Other secondary conditions may reflect differences in the behaviors, lifestyles, and social support systems between individuals who are injured at different ages. For example, burns and skin problems in areas other than the ischium, hips, or tailbone (eg, elbows or heels) may relate to high risk behavior patterns that are less frequent among people over the age of 40 at injury. Similarly, individuals who were injured later in life may show greater acceptance of the SCI and limitations it imposes; this may underlie the lower prevalence of reported stress, boredom, and family conflicts among individuals in the oldest cohort since injury. Lastly, it is possible that being injured later in life provides a physiologic buffer that decreases the likelihood of certain conditions. This would help to explain the finding that individuals who were less than 18 at injury had over 30 times greater odds of reporting kidney stones than individuals who were 40 and over at onset (a much stronger relationship than with years lived with SCI).

\section{Implications}

There are several implications of this study for rehabilitation and public health policy. First, the association of a greater number of musculoskeletal conditions with having lived 20 or more years with SCI suggests a need for routine equipment evaluations and upgrades at regular intervals in the years and decades after the onset of SCI. Regular physical therapy and evaluations, particularly those related to range-ofmotion and chair positioning, may also help to counteract the adverse consequences of aging. Second, the apparent increased risk for fractures after having lived more than two decades with SCI also suggests a need for educational programs geared at injury prevention among people with SCI. Third, health care professionals who treat urologic aspects of SCI need to work carefully with people who are injured in their teens and early twenties to develop strategies for prevention of kidney stones. Fourth, in preparing individuals for living with SCI, rehabilitation professionals need to account for the decreased risk of certain types of conditions among individuals who are older than 40 at injury. This would help to focus energy and resources on the greatest areas of need.

\section{Limitations}

In the current study, there were several limitations related to the exploratory nature of the study and subsequent research design. First, the SCQ is a measure that is in a relatively early stage of development and will no doubt undergo further revisions. Nevertheless, because it generally asks for straightforward information, it should measure the general symptoms accurately. Second, given the exploratory nature of the study, the absence of sufficient literature from which to draw specific hypotheses and the need to balance the probability of Type I error (error that occurs when the null hypotheses is rejected, but is actually true) and Type II error (error that occurs when researchers fail-toreject the null hypothesis when it is actually false), no corrections were made for multiple tests of significance. However, for a condition to have truly resulted from a Type I error and still be identified as important, it would not only have needed to be statistically significant, but also would have had to show a clear trend of differences related to either years since injury or age at injury onset. Seven conditions were statistically significant, but did not show a clear pattern of relationship between either independent variable (most of these were explained by Type II error). Third, by focusing on whether a condition had occurred at any time since injury, this study identified the relationship between aging and the appearance of conditions at some point since injury. It is possible that the frequency, number, or severity of conditions may show a different pattern with aging. This is, essentially, a different research question. Fourth, there are sampling issues that are noteworthy by virtue of utilization of a sample that: (1) was hospital based rather than population based, (2) was highly educated (although there is no evidence for this), and (3) included few racial or ethnic minority participants. 
These factors limit generalizability, although the higher educational level likely enhanced the validity of the self-report.

\section{Acknowledgements}

This research was supported by grant 5R01HD29835-02 from the National Center for Medical Rehabilitation Research of the National Institute for Health, a supplemental award from the Office on Disability and Health of the Centers for Disease Control and Prevention, and a grant from Shepherd Center. The author would like to thank Stacy Cochran, Jennifer Coker, Samuel Jones, Sarah Lottes, Joseph Maides, Kyle Massey, Maya Sternberg, Glen Sutton and Wendy Wolf, without whose contributions completion of this article would not have been possible.

\section{References}

1 National Spinal Cord Injury Statistical Center. Annual Report. Birmingham, Ala: University of Alabama 1995.

2 Vidal J, Sarrias M. An analysis of the diverse factors concerned with the development of pressure sores in spinal cord injured patients. Paraplegia 1991; 29: $261-267$.
3 Rodriguez GP, Garber SL. Prospective study of pressure ulcer risk in spinal cord injured patients. Paraplegia 1994; 32: 150 158.

4 Anderson TP, Andberg MM. Psychosocial factors associated with pressure sores. Arch Phys Med Rehabil 1979; 60: 341-346.

5 Lamid S, El Ghatit AZ. Smoking, spasticity and pressure sores in spinal cord injured patients. Am J Phys Med 1983; 62: 300-306.

6 Heinemann AW, Hawkins D. Substance abuse and medical complications following spinal cord injuries. Rehabil Psych 1995; 40: $125-140$

7 Whiteneck GG et al. Mortality, morbidity, and psychosocial outcomes of persons spinal cord injured more than 20 years ago. Paraplegia 1992; 30: 617-630.

8 Graitcer PL, Maynard FM. Psychosocial secondary disabilities. In: PL Graitcer \& FM Maynard (eds.), First colloquium on preventing secondary disabilities among people with spinal cord injuries. US Department of Health and Human Services: Atlanta, GA: $1990 ; 71-77$

9 Krause JS. Survival following spinal cord injury: A fifteen-year prospective study. Rehabil Psych 1991; 36: 89-98.

10 Krause JS, Kjorsvig JM. Mortality after spinal cord injury: A four-year prospective study. Arch Phys Med Rehabil 1992; 73: $558-564$.

11 Krause JS, Sternberg M, Maides J, Lottes S. Mortality after spinal cord injury: An 11-year prospective study. Arch Phys Med Rehabil 1997; 78: 815-821. 